\title{
HIJAS E HIJOS DE MIGRANTES EN CHILE: DERECHOS DESDE UNA PERSPECTIVA DE INCLUSIÓN SOCIAL*
}

\author{
CHILDREN OF MIGRANTS IN CHILE: RIGHTS FROM \\ A SOCIAL INCLUSION PERSPECTIVE
}

\author{
Iskra Pavez Soto** y Caterine Galaz Valderrama***
}

\begin{abstract}
Ante la intensificación de la inmigración en Chile (Thayer 2016; Tijoux y Córdova Rivera 2015), cada vez se hace más necesario estudiar los procesos de inclusión/exclusión que están viviendo los/ las hijos/as de migrantes, visualizando sus posibilidades efectivas de ejercer sus derechos como nueva ciudadanía a nivel social. Este artículo presenta resultados de una investigación acerca de los derechos de provisión, protección y participación consagrados en la Convención de los Derechos del Niño/a, respecto de hijas e hijos nacidos en Chile de madres y padres migrantes que residen en la Región Metropolitana. Se realizó desde una metodología cualitativa y focalizada en la niñez. Sus principales resultados evidencian brechas de desigualdad en el ejercicio efectivo de derechos de estos/as especialmente en el ámbito educativo y social, que además está cruzado por la forma de consideración de la "diferencia" a partir de ejes como la procedencia nacional, la clase, el género y la racialización.
\end{abstract}

Palabras claves: Infancia, derechos, desigualdad, inclusión, migrantes.

Given the intensification of immigration in Chile (Thayer 2016; Tijoux y Cordova Rivera 2015), it is becoming increasingly necessary to study the inclusion/exclusion processes experienced by migrants' children, highlighting their effective possibilities of exercising their rights as a new citizenship. This article presents the results of an investigation about the rights to provision, protection and participation - enshrined in the Convention on the Rights of the Child (CDN)-of migrants' children in the Metropolitan Region, Chile. This study was conducted using a qualitative methodology focused on childhood. Its main results show inequality gaps in the effective exercise of rights related to the educational and social fields, which are also intersected by multiple axes by the form of consideration of the "difference" from axes such as national origin, class, gender and racialization.

Key words: Childhood, rights, inequality, inclusion, migrants.

\section{Antecedentes: contextualización acerca de la infancia migrante en Chile}

Durante los últimos años, en el mundo académico y político transnacional, ha ido aumentando el interés respecto de los procesos de inclusión que viven las hijas e hijos de familias migrantes en las sociedades de instalación. En este artículo presentamos parte de los resultados de un estudio ${ }^{1}$ pionero realizado en Chile concerniente a las hijas e hijos que nacen en el país, de madres y padres migrantes. Estas hijas e hijos son catalogados desde dispositivos teóricos como "segundas generaciones" (Portes, Fernández, Kelly y Haller 2006) ${ }^{2}$. En países que tienen mayor tradición de migraciones internacionales contemporáneas, como en Estados Unidos y en Europa, ya se han observado procesos de segregación, discriminación y exclusión del ejercicio de derechos para las personas migrantes, los que pueden alterar la cohesión social, y es amplificado por los medios de comunicación o los discursos más conservadores (Solé 2005; Nash 2005, Montenegro et al. 2011).

En Chile, la presencia e instalación definitiva de personas adultas migrantes ha tomado otro cariz en los últimos años-sobre todo por la intensificación de las migraciones Sur-Sur en la región latinoamericana; sumado a que los procesos de reagrupación familiar de sus hijas e hijos comienzan incipientemente desde el 2000 (Pavez-Soto 2012). Paulatinamente, los/las descendientes de las primeras generaciones de migrantes comienzan a ser de interés académico y político, sobre todo respecto de sus niveles de inclusión y posibilidad efectiva de ejercer derechos ciudadanos en la sociedad receptora. Aunque en

\footnotetext{
* Resultado estudio “'Segundas Generaciones’ de migrantes en Chile: Integración, derechos y Políticas Públicas”, financiado por el Gobierno de Chile.

** Universidad Bernardo O’Higgins, Centro de Estudios Políticos, Culturales y Sociales de América Latina. Región Metropolitana, Chile.Correo electrónico: iskra.pavez@ubo.cl

*** Universidad de Chile, Núcleo I+D Estudios Críticos de la Diversidad. Facultad de Ciencias Sociales. Región Metropolitana, Chile. Correo electrónico: cgalazvalderrama@uchile.cl
} 
el país aún no se registran conflictos explícitos y graves de convivencia escolar y barrial (Poblete y Galaz 2016), es preciso reflexionar, desde una perspectiva proactiva, acerca de los procesos de inclusión que están viviendo las hijas e hijos de personas migrantes que nacen en Chile, tanto para conocer sus experiencias en cuanto al ejercicio de sus derechos como para proponer líneas de acción en las políticas públicas, en aras de una efectiva consideración como nueva ciudadanía (Yufra 2013).

Hasta ahora se desconoce el número exacto de hijas e hijos que han nacido en Chile de madres y padres migrantes. ${ }^{3}$ Estadísticamente solo se puede considerar a las hijas e hijos de personas migrantes que están inscritos en los registros oficiales (de acuerdo con las visas y permisos de residencia otorgados) como "migrantes". En este sentido, según estimaciones del Departamento de Extranjería y Migración (Rojas y Silva 2016), en el 2014 residían en Chile aproximadamente 411.000 personas extranjeras; de estos el 21,6\% correspondía a población infantojuvenil migrante (de 0 a 19 años).

Es importante destacar en qué ciudades y comunas se concentran más familias migrantes viviendo en el territorio, para visualizar las condiciones de vulnerabilidad o no en que este colectivo se desarrolla. La población migrante se concentra mayoritariamente en las regiones Metropolitana (61,5\%), Antofagasta $(6,9 \%)$, Tarapacá $(6 \%)$ y Valparaíso $(5,8 \%)$ y provienen principalmente de Perú $(31,7 \%)$, Argentina $(16,3 \%)$, Bolivia $(8,8 \%)$, Colombia $(6,1 \%)$, Ecuador $(4,7 \%)$, España $(3,5)$ (Rojas y Silva 2016). Dentro de la Región Metropolitana, algunas de las comunas que tienen mayor presencia de población migrante son Recoleta, Estación Central, Independencia, Santiago, Providencia y Quilicura (Thayer 2016). Por otro lado, si consideramos que la inclusión social también radica en las condiciones en que la sociedad permite que este colectivo se inserte, es importante tener en cuenta algunos indicadores sociales que muestren su situación socioeconómica: de acuerdo con la encuesta CASEN 2013 (Rojas y Silva, 2016), el 14,8\% de la población migrante vive en situación de pobreza monetaria y el $9 \%$ en situación de pobreza extrema, pero el $25,2 \%$ viviría en condiciones de pobreza multidimensional. Además de la pobreza y las condiciones de vulnerabilidad que un sector de la población inmigrada cruza, veremos más adelante que también es importante tener en cuenta que los sistemas de inclusión se vinculan con el grado de reconocimiento social que los sujetos que conviven en un espacio poseen entre sí (Fraser 2008). Sabido es que en Chile las personas migrantes padecen situaciones de discriminación racial que ha sido registrada en diversos estudios (Tijoux y Córdova Rivera 2015). Por cierto, según datos de la Encuesta de la Fundación para la Superación de la Pobreza (2012), el 41,1\% de las personas adultas extranjeras habría sufrido algún tipo de discriminación, ya sea por su nacionalidad (quienes provienen de Perú y Bolivia son quienes más sufren este tipo de acoso) o por su color de piel (Colombia, Ecuador y Haití). Por nuestra parte, en publicaciones previas (Pavez-Soto, 2012) se ha regristrado que, de modo particular, las niñas y niños peruanos experimentan graves situaciones de discriminación y racismo en Chile como también de otras nacionalidades discriminadas por procesos de racialización y de adultocentrismo (Poblete y Galaz 2016). También como antecedente es importante tener en cuenta que en Chile aún está vigente la llamada "Ley de Extranjería" promulgada en 1975 (Decreto Ley No 1094), la que no contempla los derechos de las hijas e hijos de origen migrante. Sin embargo, ya se ha presentado un nuevo Proyecto de Ley de Migraciones acorde con la realidad migratoria e inspirada en el enfoque de derechos (Rojas y Silva 2016). Paralelamente, el país ha promulgado diversos mecanismos administrativos ${ }^{4}$ para garantizar los derechos humanos de la población infantil migrante, especialmente en los ámbitos de la salud y la educación. Por otro lado, las niñas y los niños que nacen en Chile son chilenos/as, porque en el marco jurídico opera el principio de ius solis. Esta consideración final es importante de tener en cuenta porque el estudio permitió visualizar cómo niños/as que nacen en el territorio siguen siendo considerados en una "condición social de extranjería" (Santamaría 2002) por el origen de sus padres/madres.

\section{Perspectiva teórica: inclusión social y derechos de las hijas e hijos de migrantes}

En nuestro estudio se asume una perspectiva teórica basada en la inclusión, la que es entendida de diversas formas según sea aplicada en los diferentes campos de acción, por ejemplo en la educación (Matus y Rojas 2015), el ámbito de las capacidades diversas/discapacidad o en las identidades de género. Para efectos de este artículo, utilizamos la perspectiva de la inclusión social planteada por Subirats (2004). Subirats plantea que la inclusión 
social de cualquier persona o colectivo pasaría pues, en primer lugar, por el acceso garantizado a la ciudadanía y a los derechos económicos, políticos y sociales correspondientes a la misma, así como las posibilidades de participación efectiva en conjunto con el resto de la ciudadanía. También considera la conexión y solidez de las redes de reciprocidad social, ya sean estas de carácter afectivo, familiar, vecinal, comunitario. Y finalmente la inclusión implicaría participar del espacio de la producción económica y muy especialmente del mercado de trabajo (Subirats 2004). Pajares (2005) añade que este ejercicio de derechos debe ser legal y efectivo, con acceso a los cauces de participación que ofrece la sociedad autóctona. Así, los países de recepción debieran optimizar las condiciones para que las personas migrantes puedan ejercer sus derechos sociales, pero también políticos (Solé et al. 2011).

En este sentido, como se puede suponer, la contraparte de la inclusión es la exclusión. Para efectos de nuestro estudio, entendemos que la exclusión social que sufren las personas inmigrantes es un fenómeno estructural, dinámico y multidimensional que va más allá de las carencias materiales, ya que considera otros aspectos como la desigualdad en la distribución de los recursos socialmente valorados y otros cruces interseccionales de desigualdades (Romero Bachiller 2006). Por tanto, junto con las vulnerabilidades en las que puede situarse la población inmigrante, es importante tener en cuenta "la discriminación, la estigmatización, el rechazo social o la debilidad en las redes interpersonales que contribuyen o alimentan las dinámicas de expulsión u obstaculización del acceso a determinados espacios, derechos o relaciones sociales que son el único medio para alcanzar ciertos recursos" (Subirats, Alfama y Obradors 2009: 29). Estos factores de reconocimiento son importantes, porque precisamente muchas veces en los discursos de inclusión general -por ejemplo, algunos que se dan al interior de las escuelas- se invisibilizan particularidades específicas que cruzan las poblaciones migrantes, incluso como plantean Matus y Rojas (2015), discursos de inclusión pueden también normalizar a estos grupos en un espacio social que está fuera de aquellos que implícitamente se entienden como el/la alumna o escuela normal (por ejemplo, mediante estrategias de exotización cultural) (Matus y Rojas, 2015). Entonces, pensar la inclusión implica re-pensar las relaciones de los contextos con los sujetos considerados como "distintos" y cómo estos contextos utilizan metodologías, conceptos y formas comunicativas que reifican una distinción jerarquizada.

Para efectos de llevar a cabo la descripción y la interpretación de los datos recogidos en este estudio, articularemos en términos analíticos la dimensión social y cultural de la inclusión con el concepto bidimensional de justicia propuesto por Nancy Fraser (2008), ya que ella sostiene que la redistribución (el acceso a derechos sociales y económicos) debe ir acompañada necesariamente de políticas de reconocimiento (derechos políticos y culturales); de modo que, por separado, ninguno de los dos ámbitos sería suficiente. La participación de las personas extranjeras, entendida en un sentido amplio y transversal, en los procesos de redistribución y reconocimiento, debe ser parte fundamental de la inclusión social, ya que cuando los personas que habitan un determinado territorio no acceden a este escenario en igualdad de condiciones, pueden producirse fenómenos de inclusión perversa (Sawaia 2002), es decir, que se logren insertar en los espacios que no son valorados socialmente. En ese sentido, algunos/as autores/as (Rojas, Falabella y Alarcón 2016) denuncian cómo ciertas lógicas de inclusión hacen el juego a regímenes que reifican una clasificación y estigmatización de la diferencia a partir de una supuesta normalidad homogénea, y que termina legitimando ciertas desigualdades.

En ese sentido, también es importante considerar cómo las migraciones actúan como catalizador social de muchos de los cambios que actualmente a nivel transnacional está teniendo la idea de "ciudadanía”, ya que como expone Peña (2012: 529): “[las migraciones] alteran la estructura interna, social y cultural de las sociedades de acogida, mostrando la inadecuación del modelo vigente de ciudadanía estatal-nacional a la complejidad y diversidad de las relaciones sociales y formas de vida en las sociedades actuales, y modificando la percepción que los miembros de estas sociedades tienen sobre el significado de su condición de ciudadanos".

Desde reflexiones críticas, a las que adherimos, se destaca la importancia de ver a la ciudadanía como una construcción social, política e histórica, la que se ha ido formulando, por un lado, en la relación entre soberanía del Estado y los Derechos Humanos individuales y sociales y, por otro, en el devenir de los procesos de empoderamiento de distintos sujetos que han sido excluidos del ejercicio pleno de su ciudadanía, como las mujeres, los grupos étnicos, colectivos migrantes, etc. (Muñoz 2009; 
Usher 2004; Montenegro et al., 2011). Por tanto, la ciudadanía es una categoría que constituye al ser humano como sujeto político, sobre todo considerando el conjunto de derechos civiles, políticos y sociales (Subirats 2004). De ahí la importancia de valorar los procesos de inclusión de población migrante a la luz de las posibilidades efectivas de ejercicios de derechos consagrados por el país. Por ello, y considerando la población objetivo de este estudio -niños/as chilenos, hijos/as de migrantes-, tuvimos presente a la Convención de los Derechos del Niño (en adelante CDN), la que establece que existen diferentes áreas de los derechos infantiles necesarias y complementarias para una completa realización de este sector social, clasificándose en los ámbitos de la provisión, protección y participación (Alfageme et al. 2003).

\section{Apuntes metodológicos}

Esta investigación se realizó desde la metodología focalizada en la niñez (child-focused), que garantizó el anonimato (por medio del uso de pseudónimos autoseleccionados por las niñas y los niños), el respeto de los derechos de la infancia durante el estudio (James y James 2010) y la aplicación de un protocolo ético mediante la utilización de formularios de consentimiento y asentimiento informado. Además, se garantizó en todo momento el respeto a la opinión y al estado emocional de los/ las niños/as participantes.

Como ya se adelantó, la metodología del estudio fue de carácter mixto, por un lado se realizó un análisis estadístico descriptivo de los datos de hijas e hijos que han nacido en Chile de madres o padres extranjeros (se puede consultar en Pavez-Soto 2017b) y, por otro lado, se aplicó una metodología cualitativa. En este artículo se muestra solo una parte de los resultados cualitativos. Se aplicaron dos técnicas de recolección de datos: observación no estructurada de los espacios en que se desarrollan los/las niños/as para visualizar las condiciones en que estos/as se vinculan a la ciudad y entrevistas semiestructuradas en profundidad, para captar directamente sus puntos de vista y experiencias vividas.

Los sujetos participantes de la muestra del estudio debían cumplir con criterios establecidos por la investigación. Este tipo de muestra se denomina intencionada u opinática-estratégica (Valle 2003). Los criterios eran: ser niñas, niños y adolescentes de entre 8 a 17 años de edad, hijas e hijos nacidos en Chile de madres y padres migrantes. El principal criterio de exclusión era el lugar de nacimiento, porque el objetivo del estudio era, justamente, analizar a esta población (sobre niñas y niños migrantes nacidos en sus países de origen ya hemos investigado anteriormente, por ejemplo, ver Pavez-Soto 2012, 2017a; Poblete y Galaz 2016). El número de casos fue de 10 , debido a que era un estudio intensivo de datos y acotado.

Respecto de la edad, así como se muestra en la Tabla 1 (ver en Anexos), para este estudio solo pudimos entrevistar a niñas y niños de entre 8 a 13 años de edad. Durante el tiempo que realizamos el trabajo de campo pudimos lograr aplicar instrumentos de recolección de datos a niñas y niños provenientes de familias peruanas y, en menor medida, a ecuatorianas. Esta situación la corroboramos con los datos sociodemográficos de los flujos migratorios internacionales contemporáneos, los que muestran que Perú es el principal país de origen de las personas migrantes en Chile, además de ser un grupo social que lleva más años de asentamiento en el país; Ecuador aparece en menor medida (Rojas y Silva 2016). Por tanto, las hijas e hijos nacidos en Chile de familias peruanas y, en menor medida, ecuatorianas, ya tenían la edad requerida para participar en este estudio, a diferencia de niñas y niños provenientes de otros flujos migratorios más recientes.

El último criterio de la muestra era que la comuna de residencia de la Región Metropolitana fuera con altos registros de nacimientos de hijas e hijos de personas migrantes (este era un dato relativo, no absoluto, obtenido en el Registro Civil, más detalles en Pavez-Soto 2017b), como Santiago, Independencia, Recoleta, Conchalí y Estación Central; territorios que históricamente han acogido a las comunidades migrantes. Además, estos espacios sociales presentan características de vulnerabilidad socioeconómica y desigualdades, donde se intensifica la acción pública (Margarit y Bijit 2014).

\section{Resultados: las posibilidades de inclusión de hijos/as de madres/padres migrantes}

En este apartado presentaremos los resultados de las entrevistas realizadas, que nos ayudan a comprender la efectividad o no de la realización de los derechos consagrados en la Convención de Derechos del Niño en relación con los/las hijos/ 
as de madres/padres migrantes. Para efectos analíticos, en este artículo consideraremos como derechos sociales y de redistribución igualitaria, al ámbito de la provisión (derecho al nombre y la nacionalidad del Art. 7 Convención de Derechos del Niño; Derecho a la salud del Art. 24 y 39 de CDN; derecho a un nivel de vida adecuado del Art. $27 \mathrm{CDN}$; derecho la educación del Art. 28 y $29 \mathrm{CDN}$ ); mientras que como derechos culturales (reconocimiento e identidad), la protección y participación (derecho a estar protegidos de la discriminación del Art. 2 CDN).

\section{Derecho a un nivel de vida adecuado (Art. 27 CDN)}

Existe cierto consenso hoy entre quienes abordan las implicancias de los procesos migratorios que la falta de adecuación contextual para la llegada de personas migradas puede conllevar, en algunos casos concretos, vulnerabilidad (Galaz y Rubilar 2017; Stefoni et al. 2010, Pedone, Agrela y Gil 2012). El cruce de factores como la procedencia nacional, el estrato socioeconómico, el género, la edad, entre otros, junto con la falta de políticas cotidianas de inclusión efectiva, en efecto, puede llegar a constituir un factor de riesgo que se expresa de múltiples formas y que puede acompañar a algunos sectores migrantes en su instalación en las sociedades de acogida, como la chilena (Galaz, Poblete y Frías 2017). En ese sentido, las condiciones que brinda el contexto afecta de manera diferenciada a las personas migradas y sus familias. Más específicamente, la vulnerabilidad puede generarse como una condición producto de los procesos de cambio y transformación social donde se cruzan estas variables de diferenciación antes señaladas (Galaz y Yufra 2016). Tiene expresión en los niveles económicos y de bienestar de la población, como en los niveles de organización social, educación, características socioculturales, su localización en el territorio, en el manejo del medio ambiente, así como en las capacidades para recuperarse y de su adecuación al medio y a los peligros que este presenta (Rojas 2009).

Por tanto, lo que vemos en el trabajo de campo desarrollado es que las condiciones de vulnerabilidad en que se sitúan algunos/as padres y madres migrantes afectará directamente a que las niñas y los niños puedan o no ejercer su derecho de disponer de un nivel de vida digno -entendido según la
CDN-, como el derecho a una cantidad mínima de alimento, ropa y vivienda con tal de promover un desarrollo físico, mental, espiritual, moral y social. En el estudio, las niñas y los niños se plantean críticamente respecto de las formas en que viven y los espacios sociales donde habitan. En ese sentido, en el trabajo de campo se constató, mediante observaciones no estructuradas, que estos barrios están ubicados en sectores considerados como "zonas vulnerables" dentro de las comunas participantes del estudio, lo que evidencia el surgimiento de un proceso de segregación y guetificación que va configurando la identificación social de "barrios de/para migrantes".

Por otro lado, las condiciones materiales de la vivienda son bastante paupérrimas, lo que se evidencia en el hacinamiento que ya se ha identificado en otros estudios anteriores (Pavez-Soto 2012, 2017a; Liberona 2012). El hacinamiento es una situación que vulnera gravemente los derechos de las niñas y los niños, porque el habitar en escenarios de precariedad habitacional implica que otros derechos como el descanso, el juego y el nivel de vida están siendo vulnerados. En la siguiente cita podemos visualizar las condiciones antes señaladas:

"La casa la arrendamos. Vivo yo con mis tres hermanitas y mi hermano mayor, mi papá, mi mamá y, así, en una pieza vivimos, esa pieza está dentro de una casa donde viven con más familias, o sea, con tu familia, cierto. Arrendamos dos cuartos, en un cuarto yo duermo con mi hermano mayor y en el otro duerme mi mamá, mi papá y mis tres hermanas. Siete en dos habitaciones. El baño, hay dos baños, uno de hombres y otro de mujeres, así, para que no se infecte. La cocina, tenemos una cada sola, parece que hay como... parece, en cada pieza vive una familia... así a Maipú, a la Florida, partes, así, a diferentes puestos y había un puesto donde había circo gratis y juegos gratis y yo dije que quería ir allá y dijeron, es que allá no se vende y nos fuimos a otra parte. De martes a sábado, después del colegio, la ayudo a vender, sí, me gusta ayudar a mi mamá” (John, 13 años, Santiago, mamá y papá ecuatorianos).

Sin embargo, pese a estas condiciones de vivienda de algunas familias inmigradas, algunos/ 
as niñas y niños valoran positivamente disponer de una residencia y particularmente algunos espacios públicos donde juegan, así lo ejemplifica la siguiente cita:

"El barrio es espacioso, bueno y cerca de ahí no roban. Pero, la otra vez, vimos como que asaltaron a un hombre, cerca de ahí, nosotros llegando del estadio (...) Arrendamos un departamento grande, tiene un portón negro, vivimos en el piso segundo. Me gusta que tiene espacio para jugar, pero cuando descargan, tenemos poco espacio para jugar (...). A veces ayudo a transportar las cajas de abajo hasta la casa" (Batman, 9 años, Recoleta, mamá chilena, papá peruano).

\section{Derecho a la no discriminación (Art. 2 CDN)}

Algunos/as de los/las niños/as entrevistados/ as sienten que como nacieron en Chile y, por lo tanto, tienen la nacionalidad chilena, es un factor que les protege de situaciones donde podrían sufrir de prácticas discriminatorias o racistas. Esto se expresa en las siguientes citas:

"Mi papá es extranjero, pero yo me siento bien” (Redex, 11 años, Recoleta, mamá chilena, papá peruano).

"No me han molestado porque mis papás son de otro país, no, nunca. Al final, todos son peruanos" (Diana, 8 años, Conchalí, mamá y papá peruanos).

No obstante, no están exentos de ser racializados/as en algunos casos por su color de piel o hipersexualizados por las características corporales y sus estilos de habla (Galaz y Poblete 2017). En las entrevistas se aprecia que las niñas y los niños sufren situaciones de discriminación por su fenotipia y sus estilos de habla, es decir, por "parecer" migrante, aunque tengan la nacionalidad chilena. Por tanto, vemos que estamos ante procesos de extranjerización social y cultural (Santamaría 2001), cuando se traspasan características estereotipadas asociadas a unos colectivos migrantes a su descendencia. Es interesante notar la situación que comenta una niña acerca de cómo los comerciantes chilenos discriminan a los comerciantes ecuatorianos por su condición migrante (apelando en su descripción a los procesos de racialización, de desprecio etnocéntrico por los países de origen y discriminación por rasgos culturales). Esto da cuenta de las prácticas de competencia desleal y de subalternización (Galaz y Yufra 2006). Lo vemos en las siguientes citas:

"En el trabajo más, cuando trabajan como comerciantes ambulantes en la calle, a veces, no mucho, porque hay algunos chilenos que no les gusta que trabajen, así, los ecuatorianos en la calle, pero en otros lados sí, los han tratado bien, mis papás no dicen nada, se quedan, no hacen nada, los chilenos les dicen, por ejemplo, les dicen así: ustedes no son de acá, los discriminan, por el color de piel, los discriminan, a veces, los amenazan con pegarles o tirarles el paño para que se vayan de donde están trabajando, son vendedores ambulantes (...). Al principio, no me gustaba, porque cuando empecé acá, todos me miraban raro, así como que ¿quién es? y también sufrí algunas discriminaciones en todos lados, por el color de piel, me discriminaban, porque cuando iba con mi mamá, a veces, con mi mamá bajita, nos discriminaban por el porte, le decían, vieja chica, algo así le decían, así, los que pasaban por la calle, los medios flaites, creo, que son así los chilenos, yo no hacía nada, porque qué podía hacer, nada. Mi mamá tampoco hacía nada, los ignoraba no más, no les decía nada" (Flor, 12 años, Santiago, mamá y papá ecuatorianos).

\section{Derecho al nombre y nacionalidad (Art. 7 CDN)}

Debido a que el principal criterio de inclusión de la muestra del estudio era haber nacido en Chile, la totalidad de los casos entrevistados (10) tenía la nacionalidad chilena (es necesario explicitar que ninguno estaba en condición de "Hijo de extranjero transeúnte"). Como ya se dijo, en Chile rige el principio de ius solis, lo que permite que las niñas y los niños nacidos en territorio nacional tengan derecho a la nacionalidad (UNICEF 2012). Esto, como vemos en los extractos siguientes, es valorado positivamente por los/las niños/as entrevistados/as: 
"Estoy inscrita en el Registro Civil de Chile, porque tengo Carnet de Identidad" (Xiomara, 10 años, Independencia, mamá y papá peruanos).

"Yo tengo el carnet chileno, sî" (John, 13 años, Santiago, mamá y papá ecuatorianos).

Sin embargo, como vimos anteriormente, la discriminación se presenta en relación con una extranjeridad social y cultural (Santamaría 2002): es decir, que pese a tener esta nacionalidad jurídica, se manifiesten actos discriminatorios de corte racista por el hecho de ser hijo/a de personas inmigrantes, o por la fenotipia y expresión de rasgos culturales (formas de ocio, formas de habla, vestimentas, etc.). Por tanto, adquirir la nacionalidad es un paso para poder entrar al sistema social y económico, pero no asegura que puedan desarrollarse actos de división y construcción de una otredad totalmente diferente a lo considerado como "nacional" (Galaz, 2009).

\section{Derecho a la salud (Art. 24 y 39 CDN)}

Un aspecto clave en las condiciones sociales de vida se refiere al derecho de las niñas y los niños a la salud, establecido en el Art. 24 y 39 de la CDN. Las niñas y los niños están informados de los sistemas de salud a los que están adscritos ellas y ellos y/o sus familias. En general se atienden en los centros de salud públicos (CESFAM) que están ubicados cerca de sus lugares de residencia. Acerca de este ámbito, la visión crítica expuesta en las entrevistas respecto del sistema de salud se relaciona con los largos tiempos de espera para acceder a una atención, lo que se da en estos servicios públicos (Cabieses et al. 2017).

"Estoy en el Cruz Melo, tenemos Fonasa A. Casi nunca me enfermo, así que no voy tantas veces al consultorio. Cuando fui, tengo que esperar mucho, porque hay personas, hay niños menores, que ellos tiene más urgencia de lo que yo tengo y a ellos lo hacen pasar más rápido que a mí" (Xiomara, 10 años, Independencia, mamá y papá peruanos).

"A veces puedo ir a la Clínica Dávila y a veces cuando no tienen hora ahí o tarda mucho vamos al [Hospital] Roberto del Río. Tenemos FONASA, sí. El trato ahí con la gente es bueno, los tratan bien y no hay problema en eso, a los niños migrantes los tratan normales, como a toda la gente (...). Es que se demoran con algunos pacientes" (Redex, 11 años, Recoleta, mamá chilena, papá peruano).

"A un hospital o, a veces... un día me lastimé la espalda y también me fracturé el pie y me fui al [Hospital] Roberto del Río, ahí estoy inscrito. También hay un hospital de Recoleta, que se llama Recoleta de Niñas y Niños y Adultos. Yo tengo sistema público. Me atendieron bien. También, yo cuando me lastimé la espalda, más bien... sí, la espalda, estábamos esperando y nos tomó como media hora esperar a que me llamaran. No habían personas, como poquita, pero se demoraban mucho" (Jean, 9 años, Recoleta, mamá y papá peruanos).

No existen mayores dificultades para acceder a los servicios de salud primaria próximos a sus domicilios, ya que estos por lo general reciben a la población inmigrante y sus familias, principalmente a causa de que en que ciertas comunas de la Región Metropolitana han ido especializando una atención con cierto énfasis intercultural precisamente por la alta presencia de familias inmigrantes. Aunque se reconocen dificultades y muchas veces obstáculos a nivel de la atención secundaria.

\section{Derecho a la educación ( 28 y 29 de la CDN)}

Pese a los grandes avances en accesibilidad y cobertura de la población migrante en educación (Joiko y Vasquez 2016), se mantienen mecanismos informales que obstaculizan el tránsito de niños/ as migrantes e hijos/as de migrantes, actuando prioritariamente en estratos socioeconómicos más vulnerables (Stefoni et al. 2010). Las niñas y los niños entrevistados en este estudio señalan que la escuela es muy importante para ellos/as, como un espacio social, es allí donde las niñas y los niños experimentan cotidianamente los procesos de inclusión cultural a la sociedad chilena. En el ámbito escolar, por tanto, es donde señalan los/las entrevistados/as llegan a sentir actos discriminatorios o de distinción cultural con la población chilena. Como plantea Jhonson (2015), en el ámbito escolar los referentes culturales de las personas inmigradas son parte de un cierto "currículum nulo", es decir, que emerge fuera de lo que "la escuela enseña". 
De esta manera, la diferencia encarnada en los cuerpos de niños/as migrantes se hace más evidente y se culturiza y racializa. Así, las niñas y los niños nacidos en Chile de madres y padres migrantes viven la discriminación como un asunto de injusticia, a diferencia de niñas y niños que no nacieron aquí (Pavez-Soto 2012, 2017a), ya que no son discriminados por su nacionalidad legal, sino por el color de su piel y su fenotipo, por sus formas de hablar, por manifestación de formas culturales del origen de sus madres/padres, es decir, por "parecer" migrantes, tal como ya se había comentado en el apartado conceptual. Asimismo, como se ve en otras investigaciones, llama la atención la normalización que se hace de ciertos eventos, al señalar que "es normal" entre niños/as o que es la forma de "molestar" que se suele dar entre iguales (Poblete y Galaz 2016). En ese sentido, la escuela intensifica un proceso de sobreculturización y racialización que socialmente está presente en la sociedad chilena, por tanto "determinadas cuestiones sociales, económicas o propiamente escolares (y estas en interrelación con las dos anteriores) son reducidas a dificultades y soluciones étnico-nacionales" (Cucalón 2015). No obstante, en algunas escuelas se desarrollan intervenciones tempranas para evitar que estos sucesos puedan convertirse en una suerte de bullying por el origen familiar de los/las niños/ as escolarizados. Estos procesos se sintetizan en los siguientes extractos:

"En el colegio le ponen las normas, que es cosa de no molestarse, así, fuerte. Tal vez, se puede molestar, así, en forma de juego, pero, que no se pase más de eso. Cuando los niños se pasan, se ponen a pelear, la mayoría de las veces o cuando no se pongan a pelear, uno de ellos acusa y dice que lo están molestando, ahí hablan con una señorita en su oficina y resuelven el problema y llegan siendo amigos. Con los profesores bien, no nos faltan el respeto y nos tratan bien, siempre nos motivan en cosas" (Redex, 11 años, Recoleta, mamá chilena, papá peruano).

"Me siento bien, pero, hay veces, mis compañeros, mucho me molestan, al decirme que, en vez de ser chilena, parezco que soy peruana, por el color de mi piel y me discriminan, a veces. Un poco, por el color de mi piel. Me dicen que soy muy negra, pero, al compararme con ellos, soy el mismo color que ellos, que, en vez de parecer chilena, parezco blanca, por la piel, que soy más morena y los chilenos son blancos. En verdad me siento bien, porque siempre me dicen lo mismo y ya estoy acostumbrada a lo que me dicen. No está bien, no, porque en muchos casos, yo le he dicho a la directora, pero lo suspenden, pero es lo único que hacen, pero no le llaman a los padres de los niños que me molestan, pero, los que molestan son los hombres" (Xiomara, 10 años, Independencia, mamá y papá peruanos).

"En el colegio no hacía nada ¿Qué voy hacer? Bueno, a veces, los inspectores sabían hacer algo, pero no, ahora, ya no. Hasta cuando llegué a primero sí, ahora ya no, ahí no hacía nada. Cuando llegué primero a la escuela, no, no hacía nada, no. Los miraba nada más y ahora no, ahora, ya hago algo. No me gusta que me molesten, pero ya no me molestan. Yo misma les respondía, así, de lo que ellos me estaban diciendo, con cosas que a ellos les molestaba que les dijeran y se quedaban callados. Al principio de año, no fue una buena experiencia, pero ahora sí, sí, ya no es lo mismo que antes, ahora ya puedo estar más tranquila, puedo estar andando, antes no, antes me daba miedo que me molestaran y ahora ya no, ahora ando tranquilamente (...). No sé por qué me molestan... Bueno, más me molestan, hay un niño colombiano en mi curso, ese me molesta más, él molesta más. Porque yo dije una vez que yo soy chilena y él me dijo, tú andas negando a tu país, algo así, me dijo. No, yo le dije al profesor y el profesor me dijo no, no es que esté negando su país, si no que a ella le... Es que habían venido a preguntar quiénes eran chilenos y yo alcé la mano y él empezó a decir eso no más" (Flor, 12 años, Santiago, mamá y papá ecuatorianos).

\section{Derecho al juego, el descanso y la recreación (Art. 31 CDN)}

Debido a las condiciones de precariedad habitacional en que viven las niñas y los niños entrevistados, ya descritas anteriormente, en esos 
espacios se ve difícil que puedan ejercer su derecho al juego, la recreación y el descanso, establecido en el Art. 31 de la CDN. En las entrevistas pudimos apreciar que en esos espacios se ven dificultadas las posibilidades de poder jugar libremente, porque suceden procesos de externalización de la discriminación culturalista de sus madres/padres. En otras palabras, se observa que a algunas vecinas y vecinos no les agrada convivir con personas inmigradas, por lo que molestan a los/las niños/as como una forma de presión para que las familias migrantes se muevan del lugar residencial compartido:

"El parque no está tan cerca de acá. Cuando juego acá, juego en el estacionamiento que hay abajo" (Redex, 11 años, Recoleta, mamá chilena, papá peruano).

"En el barrio, el espacio que hay para jugar, es, si, eh afuera, en la calle, plaza Vivaceta, voy los domingos, juego en el resbalín, con mis vecinos" (Antonella, 10 años, Independencia, mamá peruana, papá chileno).

"En mi casa vamos a jugar a la pelota, en la plaza, en el edificio, no nos dejan hacer bulla, para nada. Que en la noche hay un espacio, un poco de espacio, donde salgo con mi papá, y mis amigos, mis papás me vigilan y yo juego con mis amigos peruanos y chilenos, de doce, o sea de diez a catorce años, la mayoría de catorce años. A veces, salgo solo, pero como hay carabineros, estoy protegido. Jugamos al pillarse y a la escondida" (Roro, 12 años, Santiago, mamá y papá peruanos).

"Afuera solo los domingos o casi en la noche, cuando ya no hay gente afuera, salimos un rato a jugar, afuera, así, en la calle, en la cuadra, en la vereda. Con niños más chicos que yo, sí, como de siete años, siete, ocho, hasta diez años. Son ecuatorianos. Nosotros salimos con bicicletas, salimos a hacer carreras, así, con patinetas, así, cosas o cuando jugamos a la pelota, afuera. Pero la cuadra es ancha, así que, sí, si pasan autos, pero ya en la noche, ya no pasan. Les gusta jugar un juego que se llama carnaval, en el patio de atrás y eso a los que viven arriba no les gusta, a algunos, a una familia que vive arriba no les gusta, porque dicen que moja mucho el piso o que desordenan las cosas. Ya, entonces, por eso se enojan o porque a veces hacen cumpleaños, así, o fiestas en la misma casa y dicen que hacen mucha bulla. No les gusta" (Flor, 12 años, Santiago, mamá y papá ecuatorianos).

Si bien las niñas y los niños pueden ejercer su derecho al juego, descanso y recreación en otros espacios como la escuela o centros comunitarios, es de vital importancia considerar los obstaculizadores que encuentran en los territorios donde habitan cotidianamente y donde se deberían llevar a cabo los procesos de inclusión con los otros actores locales, así como hijas e hijos de madres y padres chilenos, vecinas y vecinos adultos, etc. Lamentablemente, en las entrevistas también se observa que cuando una zona es sobrehabitada por determinados grupos sociales se estigmatiza a todo el colectivo y se estereotipa un territorio desde una posición etnocéntrica y diferenciadora: por ejemplo, en un relato encontramos el caso de un espacio público que comienza a ser percibido por la comunidad local como "El parque de los peruanos":

"Es que cerca íbamos a jugar a la pelota, hasta que se incendió un auto, y ya saben, mi papa llegó con su extintor y se acabó rápido, llegaron los bomberos y todo eso. Ahí hay como un parque, sí, muchos parques, solo que, a veces, hay un parque entero, grande, pero, están en distintas, están separadas" (Batman, 9 años, Recoleta, mamá chilena, papá peruano).

"Juego en el parque, en el de... que acá le dicen "El parque de los peruanos", es un parque común y corriente, que queda por Rivera [risas]. Pero tengo una amiga chilena que va allá, entonces, no entiendo por qué le dicen así, a ese parque. A veces, mi mamá se comunica con una señora, que también es peruana y su hija chilena, como yo y nos juntamos en el parque y con ella juego, a veces, encuentro mi mejor amiga que se llama Aldana y también ella es peruana, sí, ella si es peruana y con ella jugamos, a veces. A partir de las cinco me voy al parque, me quedo hasta como las ocho. Me gusta el columpio, que está ahí, también hay juegos inflables, que me gustan y esas máquinas para hacer ejercicios" (Xiomara, 10 años, Independencia, mamá y papá peruanos). 
"Nos vamos a la plaza, por cerca de Santa Isabel, no sé cómo se llama, hay juegos, a veces, jugamos al futbol y, si no traemos pelota, en los columpios. Nos vamos, todos los chicos... con mis primos chiquitos, mi hermana, mi hermanita chiquita, mi hermano" (John, 13 años, Santiago, mamá y papá ecuatorianos).

\section{Conclusiones}

Los datos cualitativos de este estudio nos permiten poner en cuestión las maneras en que Chile está disponiendo acciones que incidan en una inclusión efectiva para la población inmigrante, lo que inevitablemente redunda en cómo se insertan social y culturalmente actualmente sus hijos/as; sobre todo la generación nacida aquí en Chile. Vemos que en las opiniones que realizan algunos/as niños/as se pone en cuestión la consideración de ciudadanía universal, precisamente porque sus comentarios aluden al establecimiento de una diferencia jerarquizada (Anthias 2006): es decir, la distinción entre nacionales y extranjeros/ as no está supeditada al acto legal de disponer la nacionalidad en sí misma (como es su caso), sino en consideraciones culturales y racializadas. Por tanto, pese a tener acceso a los mismos derechos que los/as niños/as chilenos/as de padres/madres chilenos/as, los/las hijos/as nacidos en Chile de migrantes viven una encarnación de características de sus grupos de origen que los sitúa, nuevamente, en construcciones de otredad. Siguiendo la lógica de inclusión planteada por Subirats (2004), estos/ as niños/as nacidos en el país disponen de ciertos derechos económicos, políticos y sociales; sin embargo, su valoración social (reconocimiento dirá Fraser, 2008) no se sitúa en igualdad de condiciones, ya que son considerados/as como otros/as, a partir de una extranjerización social y cultural. También los relatos de los/las niños/as nos muestran que sus redes de reciprocidad-factor también importante al considerar una efectiva inclusión- tiene altos y bemoles: disponen de condiciones afectivas familiares, pero muchas veces entran en crisis las relaciones vecinales y comunitarias. Por otro lado, si bien estos/ as niños/as comparten la vulnerabilidad propia de ciertos barrios sociales ubicados en los escalafones socioeconómicos menos apreciados socialmente en la Región Metropolitana-lo que sucede también con parte de la población chilena-; su vulnerabilidad se acrecienta al pertenecer a familias con padres/ madres inmigrantes que son vehiculizados solo a ciertos nichos residenciales y bajo ciertas condiciones de habitabilidad (Margarit y Bijit 2004), lo que les termina afectando en sus condiciones de vida y el efectivo ejercicio de derechos como lo vimos al ejemplificarlo por medio de la Convención de Derechos del Niño. Los/las niños/as destacan en las entrevistas en particular los derechos al ocio, el juego y el derecho a la ciudad.

Asimismo, en nuestra investigación se comprobó que algunas familias migrantes se insertan en los estratos más precarizados de nuestra sociedad, lo que repercute en que tanto madres/padres migrantes como sus hijos/as acceden a servicios de educación y salud de baja calidad y a espacios públicos poco equipados. Por tanto, como señala Sawaia (2002), están dentro del sistema pero en los espacios donde nadie desea habitar. En ese sentido, en los relatos expuestos por los/las niños/as se vinculan interseccionalmente (Romero Bachiller 2006) diferentes ejes de desigualdad como la edad, la clase, el origen étnico, los rasgos fenotípicos y la procedencia nacional corporizadas desde sus padres/madres, haciendo que los procesos de vida de niños/as hijos/as de migrantes tengan características específicas actualmente que los sitúa en espacios de otredad; lo que es preocupante respecto de una efectiva inclusión ciudadana en igualdad de condiciones. Por tanto, se requiere estudiar estas encrucijadas de factores, ahondar en esas prácticas que racializan y que culturalizan a generaciones que no necesariamente son migrantes de hecho.

Es necesario considerar que actualmente en Chile se están discutiendo reformas políticas claves: por ejemplo, la Reforma Educacional, la creación de una Nueva Institucionalidad de Infancia y un nuevo Proyecto de Ley de Migraciones. Por tanto, las propuestas que defiendan los derechos de las hijas e hijos nacida/os en Chile de madres y padres migrantes pueden ser un elemento aportador en el debate social respecto de la construcción jerarquizada de diferencias que estamos presenciando. Asimismo, los resultados del estudio muestran la necesidad de generar políticas sociales universales y focalizadas que consideren las necesidades también de las familias migrantes como nuevos sujetos políticos y ciudadanos. Como plantea Tubino (2005), el problema de fondo no es cómo descentralizar al Estado-Nación homogeneizador, cómo simplemente ampliar su cobertura social o hacerla más eficiente, 
sino preguntarse si el Estado que tenemos es el que se necesita considerando los cambios contextuales y la diversidad ciudadana que nuestros países están construyendo y la interculturalidad que se vive cotidianamente. Precisamente la migración permite poner en tela de juicio el modelo de Estado-Nación y avanzar a prácticas que unan derechos universales con derechos particulares, involucra, en definitiva, pensar un nuevo modelo de Estado (Tubino 2005).

Durante los últimos años las familias chilenas han experimentado cambios en las formas de vivir posibilidades de inclusión/exclusión en el Chile contemporáneo: hoy la pobreza no es solo la falta de ingresos económicos; de hecho, se ha introducido una nueva forma de medición -la Pobreza Multidimensional-, incorporando variables como salud y educación. En este escenario, las familias migrantes quedan situadas en condiciones de alta precariedad y vulnerabilidad con características particulares que las diferencian de las familias chilenas del mismo estrato social, lo que las hace merecedoras de ser consideradas de una forma específica en las escasas prestaciones del Estado. Todo ello, pensando en cómo también cualquier sistema de priorización puede generar rechazo en la comunidad chilena, aumentando los riesgos de hostilidad frente a estas $y$, por tanto, las posibilidades de ser víctimas de discriminación y racismo. Los servicios o bienes a los que tienen acceso las niñas y los niños nacidos en Chile de madres y padres migrantes no deben ser, necesariamente, distintos a los ofrecidos para las hijas e hijos de madres y padres chilenos. Se trata, más bien, de que las políticas públicas se diseñen, ejecuten y evalúen a partir de estándares de calidad que sean universales, basadas en el enfoque de derechos. Estas políticas públicas universales se inscriben principalmente en el área de los Derechos de Provisión. De igual modo, es necesario que existan algunas actuaciones focalizadas que atiendan la particularidad de las niñas y los niños que nacen en Chile de madres y padres de origen migrante. Se trata de Políticas de Reconocimiento (Fraser 2008) -inscritas en el ámbito de los Derechos de Protección y Participación-para afectar de mejor manera las discriminaciones, los procesos de racialización que están viviendo muchos/ as niños, pese a no ser migrantes de hecho (ya que al haber nacido aquí, no han vivido procesos de movilidad).

Por tanto, avanzar hacia una inclusión social de estos nuevos sujetos residentes a largo plazo implica pensar políticas públicas que pongan el acento en la gestión más próxima de las personas pero considerando no solo el eje socioeconómico sino también la noción de reconocimiento y de igualdad de oportunidades. De allí que las políticas públicas tomen un peso importante, ya que estas inciden directamente en las posibilidades efectivas de inclusión social de las niñas y niños al desarrollar o reproducir actuaciones y discursos en cuanto al reconocimiento enunciativo, la incorporación sociocultural y la igualdad de oportunidades de este colectivo (Galaz, Poblete y Frías 2017). Esto permite avanzar en el reconocimiento de que ciertos sectores de la población requieren la adopción de medidas especiales de equiparación con los otros sectores más aventajados, especialmente considerando los hijos e hijas de personas inmigrantes.

En definitiva, asegurar los derechos a las hijas e hijos de migrantes implica re-pensar los límites de lo que se considera como "ciudadanía". Así, las políticas públicas deberán incidir en las diferentes dimensiones interdependientes que afectan a los miembros de una sociedad, así como su situación económica, social y cultural, garantizando los derechos individuales y sociales de sus miembros y reconociendo la complejidad y diversidad de la sociedad. En esa diversidad se inscribe la población migrante, pero aún más las generaciones que sin ser migrantes se les trata como tal. Re-pensar la ciudadanía nos invita a centrarnos en qué derechos civiles, políticos y sociales se están actualmente jerarquizando a partir de procesos de diferenciación social, solo por el hecho de ser extranjeros/as, y cómo esas jerarquizaciones están afectando la vida de niños/as -nacionales y extranjeros-alejándose de los derechos garantizados internacionalmente a estos/as.

\section{Agradecimientos}

Expresamos nuestro agradecimiento al Departamento de Extranjería y Migración (DEM) dependiente del Ministerio del Interior y Seguridad Pública del Gobierno de Chile, por haber financiado el estudio " 'Segundas Generaciones' de migrantes en Chile: Integración, derechos y Políticas Públicas", coordinado por la Dra. Iskra Pavez-Soto, mediante la Convocatoria 2016 de Trato Directo: "Servicio personal especializado consistente en la elaboración de un estudio sobre migraciones". Resolución Exenta N ${ }^{\circ} 5673$, del 29 de septiembre de 2016. 
Anexo

Tabla 1. Antecedentes sociodemográficos de hijas e hijos nacidos en Chile de madres y padres migrantes participantes del estudio

\begin{tabular}{llccc}
\hline \multirow{2}{*}{ Comuna de residencia } & \multicolumn{1}{c}{$\begin{array}{c}\text { Pseudónimo } \\
\text { autoseleccionado }\end{array}$} & Edad & Nacionalidad madre & Nacionalidad padre \\
\hline \multirow{2}{*}{ Recoleta } & Jean & 9 & Peruana & Peruano \\
& Redex & 11 & Chilena & Peruano \\
& Batman & 9 & Chilena & Peruano \\
\hline \multirow{2}{*}{ Independencia } & Xiomara & 10 & Peruana & Chileno \\
& Antonella & 10 & Peruana & Peruano \\
\hline Conchalí & Diana & 8 & Peruana & Ecuatoriano \\
\hline \multirow{2}{*}{ Santiago } & Jhon & 13 & Ecuatoriana & Ecuatoriano \\
& Roro & 12 & Ecuatoriana & Ecuatoriano \\
\hline Estación Central & Flor & 12 & Ecuatoriana & Peruano \\
\hline
\end{tabular}

\section{Referencias Citadas}

Alfageme, E.; Cantos, R. y Martínez, M.

2003 De la participación al protagonismo infantil. Propuestas para la acción. Plataforma de Organizaciones de Infancia. Madrid.

Anthias, F.

2006 "Género, etnicidad, clase y migración: inteseccionalidad y pertenencia transnacional". En P. Rodríguez (ed.), Feminismos periféricos. Ed. Alhulia. Granada.

Cabieses, B.; Bernales, M. y McIntyre, A.M.

2017 La migración internacional como determinante social de la salud en Chile: evidencia y propuestas para políticas públicas. Universidad del Desarrollo. Santiago de Chile. Disponible en: http://www.udd.cl/dircom/pdfs/Libro_La_migracion_internacional.pdf

Convención sobre los derechos del niño (CDN).

1989 Unicef. Disponible en: http://unicef.cl/web/ convencion-sobre-los-derechos-del-nino/

Cucalón Tirado, P.

2015 "A vueltas con la cultura: Imágenes del alumnado inmigrante en las aulas de enlace de la comunidad de Madrid (España)". Diálogo Andino, No 47, pp. 85-94. Chile.

Departamento de Extranjería y Migración DEM.

2016 Estadísticas Migratorias 2005-2015. Departamento de Extranjería y Migración, 2016, disponible en http:// www.extranjeria.gob.cl/estadisticas-migratorias/ [consulta 19-07-2016]

Fraser, N.

2008 "La justicia social en la era de la política de identidad: redistribución, reconocimiento y participación". Revista de Trabajo, 6, pp. 83-99. Argentina.

Fundación para la Superación de la Pobreza.

2012 Ser migrante en el Chile de hoy: Encuesta 2012. Santiago, Fundación para la Superación de la Pobreza. Chile.

Galaz, C. y Rubilar, G.

2017 Caracterización de población dominicana residente en Chile. Núcleo Estudios Críticos de la Diversidad, Santiago, Chile. Disponible en: https://www.researchgate.net/project/
Caracterizacion-de-la-poblacion-Dominicana-residente-enChile.

Galaz, C. y Yufra, L.

2016 Diferencias, jerarquías, subalternidad: discursos y prácticas de intervención hacia mujeres inmigradas. Forum Qualitative Sozialforschung / Forum: Qualitative Social Research, 17(3), Art. 19, Alemania.

Galaz, C.; Poblete, R. y Frías, C.

2017 Políticas públicas e inmigración ¿Posibilidades de inclusión efectiva en Chile? Editorial Universitaria. Santiago de Chile.

James, Allison y James, Adrian.

2010 Key concepts in Childhood Studies, SAGE, London. Jhonson, Mardones D.

2015 "Formar ciudadanos interculturales en un mundo global: algunas notas de los estudios curriculares". Diálogo Andino $\mathrm{N}^{\circ}$ 47, 2015. Páginas 7-14. Chile.

Joiko, S. y Vásquez, A.

2016 "Acceso y elección escolar de familias migrantes en Chile: 'No tuve problemas porque la escuela es abierta, porque acepta muchas nacionalidades"'. Calidad en la Educación, 45, 132-173. Chile.

Liberona, N.

2012 "De la alterización a la discriminación en un sistema público de salud en crisis: Conflictos interétnicos a propósito de la inmigración sudamericana en Chile". Revista Ciencias Sociales, 28, 31-38. Chile.

Margarit, D. y Bijit, K.

2014 "La integración social de los inmigrantes latinoamericanos en la estructura urbana". En M. Tapia y A. González (Eds.), Regiones Fronterizas: Migración y los desafíos para los Estados nacionales latinoamericanos. Págs. 309-333. Ril editores. Chile.

Matus, C. y Rojas, C.

2015 "Normalidad y diferencia en nuestras escuelas: a propósito de la Ley de Inclusión Escolar". Docencia, 56, 47-56. Chile. 
Montenegro, M.; Galaz, C.; Yufra, L. y Montenegro, K. 2011 "Dinámicas de subjetivación y diferenciación en servicios sociales para Mujeres inmigradas en la ciudad de Barcelona”. Athenea Digital, 11(2), 113-132. España.

Muñoz, J.

2009 "Derechos Humanos, Migraciones y Ciudadanía Universal”. Diálogos Migrantes (2), pp. 8-20. Colombia. Ministerio de Desarrollo Social.

2012 Resultados Encuesta CASEN 2011. Bases de datos. Nash, M.

2005 "La doble alteridad en la comunidad imaginada de las mujeres inmigrantes". En Nash Mary, Tello Rosa y Benach Núria (eds.), Inmigración, género y espacios urbanos. Los retos de la diversidad. Edicions Bellaterra. Barcelona.

Pedone, C.; Agrela, B. y Gil, S.

2012 "Políticas públicas, migración y familia. Una mirada desde el género". Papers, 97(3), 541-568. España.

Pajares, Miguel

2005 La integración ciudadana. Una perspectiva para la inmigración. Icaria. Barcelona

Pavez-Soto, I.

2012 "Inmigración y racismo: experiencias de la niñez peruana en Santiago de Chile". Si Somos Americanos: Revista de Estudios Transfronterizos, 12, 75-99. Chile.

Pavez-Soto, I.

2017a "Integración sociocultural y derechos de las niñas y los niños migrantes en el contexto local. El caso de Recoleta (Región Metropolitana, Chile)", Chungará, Revista de Antropología Chilena, Vol. 49, № 4, pp. 613-622. DOI: http:// dx.doi.org/10.4067/S0717-73562017005000105, Chile.

Pavez-Soto, I.

2017b “'Segundas Generaciones' de Migrantes en Chile: Integración, Derechos y Políticas Públicas", Informe final de estudio, Departamento de Extranjería y Migración (DEM), Ministerio del Interior y Seguridad Pública, Convocatoria de Trato Directo año 2016. http://www.extranjeria.gob.cl/media/2017/12/ DEMInvestiga1SegundasGeneracionesdeMigrantesenChile. pdf, Chile.

Pavez-Soto, I. y Chan, C.

2017 "The second-generation in Chile: Negotiating identities, rights, and public policy", International Migration Review. DOI: 10.1111/imig.12410. Estados Unidos.

Peña, J.

2012 "Migraciones y Apertura Cosmopolita de la Ciudadanía". Arbor Ciencia, Pensamiento y Cultura, 188(755), pp. 529542. España.

Poblete, R. y Galaz, C.

2007 "La identidad en la encrucijada; migración peruana y educación en el Chile de hoy". II Congreso Internacional de Etnografía y Educación, Migraciones y ciudadanía. EMIGRA. Barcelona. Universidad Autónoma de Barcelona. Publicación online: https://ddd.uab.cat/pub/emigrawp/ emigrawp_a2007n3/emigrawp_a2007n3p1.pdf

Poblete, R. y Galaz, C.

2016 Niños y niñas inmigrantes: Trayectorias de inclusión educativa en escuelas de la Región Metropolitana. Superintedencia de Educación, Ministerio de Educación. Chile.

Portes, A.; Fernández-Kelly, P. y Haller, W.

2006 "La asimilación segmentada sobre el terreno: la nueva segunda generación al inicio de la vida adulta". Migraciones, 19, 7-58. España.
Romero Bachiller, C.

2006 Articulaciones Identitarias: Prácticas y representaciones de género y "raza"/etnicidad en "mujeres inmigrantes" en el barrio de Embajadores (Madrid). Madrid: Universidad Complutense de Madrid.

Rojas, N. y Silva, C.

2016 La Migración en Chile: Breve Reporte y Caracterización. Informe Observatorio Iberoamericano sobre Movilidad Humana, Migraciones y Desarrollo. OBIMID. Madrid.

Rojas, M. T.; Falabella, A. y Alarcón, P.

2016 Inclusión social en las escuelas: estudio de prácticas pedagógicas inclusivas y proyecciones para enfrentar un escenario sin copago y selección escolar. Ministerio de Educación. Chile.

Rojas, M. T.

2009 ¿Es posible imaginar escuelas más integradas socialmente? Respuestas de directivos, docentes y familias frente a una normativa que promueve la integración social”, en Revista Docencia, No 39, Año XIV, Chile.

Sánchez-Melero, H. y Gil Jaurena, I.

2015 "Análisis interseccional y enfoque intercultural en el estudio de la ciudadanía y la participación. Consideraciones epistemológicas". Diálogo Andino. № 47. Páginas 143-149. Chile.

Santamaría, E.

2002 La incógnita del extraño. Una aproximación a la significación sociológica de la "inmigración no comunitaria". Anthropos. Barcelona.

Sawaia, B.

2002 O sofrimento etico-político como categoria de análise da dialéctica exclusão/inclusão. En Sawaia B. (Org.), As artimanhas da exclusão. Análise psicossocial e ética da desigualdade social, cuarta edición (pp. 97-118). Petrópolis: Vozes. Brasil.

Solé, C.; Sordé, T.; Serradell, O.; Alcalde, R.; Flecha, A.; Pettroff, A.; Cavalcanti, L.; Parella, S.; Pavez, I.; Santamaría, E. y Garzón, L.

2011 "Cohesión Social: aportaciones científicas y discursos políticos”. Revista Internacional de Sociología 69: 9-32. España.

Solé, C.

2005 "Sociedades de vieja y nueva inmigración”. En: Solé, Carlota y Flaquer, Lluís. (Eds.). El uso de las políticas sociales por parte de las mujeres inmigrantes. Madrid: Instituto de la Mujer-Ministerio de Trabajo y Asuntos Sociales, pp. 55-66. España.

Subirats, J.

2004 Pobreza y Exclusión Social: Un análisis de la realidad española y europea. Fundación "la Caixa". Barcelona.

Stefoni, C.; Acosta, E.; Gaymer, M. y Casas-Cordero, F.

2010 El derecho a la educación de los niños y niñas inmigrantes en Chile. Universidad de Deusto. Bilbao.

Tijoux, M.E. y Córdova Rivera

2015 "Racismo en Chile: colonialismo, nacionalismo, capitalismo". Polis. Revista Latinoamericana, Volumen 14, $\mathrm{N}^{\circ}$ 42, 2015, pp. 7-13. Chile.

Thayer, L. E.; Stang, F.; Dilla, Ch. y Durán, C.

2016 Observatorio de políticas locales: Precariedad institucional y arbitrariedad. En: Seminario Internacional Migrantes y Refugiados: Los Derechos Humanos desde los Gobiernos Locales, 18 y 19 de agosto de 2016, Quilicura. 
Tubino, F.

2005 "La praxis de la interculturalidad en los Estados Nacionales Latinoamericanos". Cuadernos Interculturales, 3(5), 83-96. Chile.

\section{UNICEF.}

2012 Los derechos de los niños, niñas y adolescentes migrantes, refugiados y víctimas de trata internacional en Chile. Avances y desafíos. Santiago: Ed. EIRL-UNICEF.

Usher, E.

2004 Fundamentos de Gestión de la Migración. Para las Personas Encargadas de Formular Políticas y Profesionales.
Organización Internacional para las Migraciones, Ginebra.

Valles, M.

1997 Técnicas Cualitativas de Investigación Social. Reflexión Metodológica y Práctica Profesional. Síntesis, Madrid. España. Yufra, L.

2013 "Inmigrantes en formación: Estado de bienestar y promoción de lazos sociales en el caso de la formación para la inserción laboral de las personas inmigrantes en Barcelona". Athenea Digital. Revista de pensamiento e investigación social, 13(2), 301-309. España.

\section{Notas}

1 El estudio “'Segundas Generaciones' de migrantes en Chile: Integración, derechos y Políticas Públicas" fue financiado por el Departamento de Extranjería y Migración (DEM). La investigación tuvo como objetivo hacer un primer diagnóstico respecto de la situación de las hijas e hijos que nacen en Chile de madres y padres migrantes. La metodología fue de carácter mixto; por un lado se realizó un análisis estadístico descriptivo de los datos de hijas e hijos que han nacido en Chile de madres o padres extranjeros desde 1990 a 2016; información solicitada mediante la Ley de Transparencia (Ley N ${ }^{\circ} 20.285$ sobre Acceso a la Información Pública, 11 de agosto de 2008) al Registro Civil e Identificación. Por otro lado se aplicó una metodología cualitativa (observación y entrevistas) a una muestra de 10 hijas e hijos nacidos en Chile de madres y padres migrantes. En este artículo se consigna solo una parte de los resultados cualitativos. El análisis de los datos cuantitativos del estudio se puede consultar en Pavez-Soto 2017b.

2 Ver debate sobre concepto de "Segunda generación" en Pavez-Soto y Chan 2017

3 Se solicitó al Registro Civil e Identificación (por medio de la Ley de Transparencia) los datos de hijas e hijos que han nacido en Chile de madres o padres extranjeros desde 1990 a 2016. Sin embargo, la información recibida presentaba graves errores, por tanto no se pudo establecer el número exacto $(\mathrm{N})$ de esta población, solo se obtuvo la distribución relativa (\%). Los datos cuantitativos están analizados en Pavez-Soto 2017b.
4 Por ejemplo, el Ministerio de Educación ha emitido algunos instructivos (Oficio Circular N ${ }^{\circ}$ 6.232-Mayo 2003, Oficio ORD N ${ }^{\circ}$ 07/1008/1531-Agosto 2005 del Ministro de Educación) para garantizar el ingreso escolar de la niñez migrante (la "matrícula provisoria" permite el pago de subvención escolar mientras se legalizan los certificados académicos). En 2007 se firma el convenio que facilita el acceso a la educación parvularia (Resolución Exenta $\mathrm{N}^{\circ} 6.677$ de Noviembre 2007). En 2008 se establece el convenio que facilita el acceso al sistema público de salud de niñas, niños y adolescentes extranjeros, independiente de su situación migratoria en Chile y la de sus madres, padres, tutores o representantes legales (Resolución Exenta № 1.914 de marzo de 2008). En 2009 se firmó el Convenio de acceso a la red de protección de SENAME para niñas y niños migrantes (Resolución Exenta $N^{\circ} 10.654$ de diciembre 2009). En 2014 DEM emitió el Oficio No 27.601 y el Registro Civil emitió la Resolución Exenta № 3.207, donde se modifica la aplicación de facto del criterio "hijo de extranjero transeúnte", establecida en el Art. 10 de la Constitución, era entendida como equivalente a irregularidad migratoria. Este criterio administrativo fue corregido en 2014, permitiendo la adecuada aplicación constitucional, y esto ha posibilitado que aquellos que habían sido inscritos bajo esta categoría pudiesen rectificar su inscripción como chilenos/as mediante el Pronunciamiento de Nacionalidad, situación que pasó de 40 en el 2013 a 102 en el 2014 (DEM 2016). 